\title{
Some topological properties of quotients modulo semisimple algebraic groups
}

\author{
R. V. Gurjar
}

\begin{abstract}
We will prove a general result in Invariant Theory, viz. for a quotient $\mathbb{C}^{n} / / G$, where $G$ is a connected complex semisimple algebraic group, the local first homology group at any point in the quotient $\mathbb{C}^{n} / / G$ is trivial and the local second homology group is finite. Using this we will prove that the completion of the local ring of any point in $\mathbb{C}^{n} / / G$ is a unique factorization domain (UFD).
\end{abstract}

Mathematics Subject Classification (2000). 13A50, 14L24, 14L30.

Keywords. Semisimple algebraic group, quotient, homology group.

\section{Introduction}

The aim of this paper is to prove the following general result in Invariant Theory.

Theorem. Let $G$ be a complex linear algebraic group such that its connected component of identity $G^{0}$ is semisimple. Suppose $G$ acts on $\mathbb{C}^{n}$ regularly and $W:=\mathbb{C}^{n} / / G$ is the quotient corresponding to the ring of invariants $\mathbb{C}\left[X_{1}, X_{2}, \ldots, X_{n}\right]^{G}$ such that $\operatorname{dim} W>1$. Then we have the following assertions:

(a) For any point $p \in W$ the local first homology group $H_{1}^{p}(W)$ is finite. If $G$ is connected then $H_{1}^{p}(W)=(0)$.

(b) If $W$ has isolated singularities then the fundamental group at infinity, $\pi_{1}^{\infty}(W)$, is trivial.

(c) The local second homology group $H_{2}^{p}(W)$ is finite. Further, $H_{2}(W \backslash$ Sing $W)$ is finite.

Remark. The proof of the second part of (c) above plays an important role in many arguments.

For the necessary topological definitions and notations, see Section 1. 
Remarks. (a) It is proved in [8] that the local fundamental group $\pi_{1}^{p}(W)$ is always finite whenever $G$ is (not necessarily connected) reductive. But even if $G$ is connected semisimple $\pi_{1}^{p}(W)$ can be non-trivial. The author is thankful to T. N. Venkataramana for showing a construction of examples of this. See remark at the end of the proof of the theorem.

(b) If $G^{0}$ is not semisimple then $H_{2}^{p}(W)$ can be of rank $>0$. An example of this is given after the proof of theorem.

We have the following useful consequence of the theorem.

Corollary. Let $G$ be a connected semisimple group and let $W, p$ be as in the statement of the theorem. Then the analytic local ring $\mathcal{O}_{W, p_{\mathrm{an}}}$ is a UFD.

The example given after the proof of the theorem shows that for $G$ connected reductive this is not always true. In the corollary, presumably even the completion of $\mathcal{O}_{W, p}$ w.r.t. its maximal ideal is factorial. The example mentioned above can be modified to show that the local ring of a rational singular point on an algebraic variety can be a UFD but its completion may not be so.

V. B. Mehta has informed the author that there exists an example of a quotient $\mathbb{C}^{n} / / G$ which has a factorial local ring but whose completion is not factorial. In this example $G$ is a torus.

Acknowledgements. The author is thankful to T. N. Venkataramana for showing some interesting representations of semisimple groups which illustrate that we cannot expect triviality of the local fundamental group of quotients in the theorem. He would also like to thank V. L. Popov for showing an example where the complement of the principal stratum in the Luna stratification can have divisors even for connected semisimple groups. The author is thankful to the referee for carefully reading the manuscript, pointing out many inaccuracies in the earlier version and making useful suggestions for improving the readability of the paper.

\section{Preliminaries}

All the varieties considered in this paper are complex algebraic or analytic varieties.

For any topological space $T$ we will denote by $H_{i}(T)$ (resp. $H^{i}(T)$ ) the singular homology (resp. cohomology) with integer coefficients.

For any normal algebraic variety or a normal complex space $Z$ and $p \in Z$ the local $m^{\text {th }}$ homology group $H_{m}^{p}(Z)$ is defined as the group $H_{m}(U-\operatorname{Sing} U)$, where $U$ is a suitable small neighborhood of $p$ in $Z$. This notion is well-defined. There are arbitrarily small such neighborhoods of $p$. One way to see this is to use a triangulation of $Z$ such that $\operatorname{Sing} Z$ is a closed subcomplex and use local properties of simplicial 
complexes (see [6]) or [17], Chapter II, §6). If $p$ is a simple point of $Z$ then we consider $H_{m}(U-p)$ for the definition of $H_{m}^{p}(Z)$. If $\operatorname{dim} Z=d$ and $p$ is a simple point then $U-p$ has the homotopy type of the sphere $S^{2 d-1}$. Similarly we can define the local fundamental group, $\pi_{1}^{p}(Z)$, for any normal complex space and $p \in Z$.

If $Z$ is affine of dimension $\geq 2$ and has only isolated singularities then the fundamental group at infinity, $\pi_{1}^{\infty}(Z)$, is defined as the fundamental group of the complement of a sufficiently large compact subset $K$ with $C^{\infty}$ boundary in $Z$. It can be calculated using a suitable compactification of $Z$ as in the proof of assertion (b) of the theorem below.

Recall that if a reductive algebraic group $H$ acts regularly on a normal affine variety $X$ then the ring of invariants $\Gamma(X, \mathcal{O})^{H}$ is finitely generated and normal. The corresponding normal affine variety is denoted by $X / / H$. The morphism $\pi: X \rightarrow$ $X / / H$ is called the quotient map. For many standard properties of the map $\pi$, see [16].

We will abbreviate by RDP a rational double point singularity of a complex analytic surface. It is well known that if $(V, p)$ is a germ of an RDP then the homology group $H_{1}(V-\{p\})$ is finite and $H_{2}(V-\{p\})=(0)$. A small open neighborhood $V$ of $p$ behaves like a topological manifold, at least when we use rational homology.

We will use the following results about rational and canonical singularities ([19]).

(1) A general hyperplane section of a variety with only rational singularities again has only rational singularities ([19], §1).

(2) The singularities of a Gorenstein variety $V$ with at most rational singularities are canonical singularities. This follows from the definition of a canonical singularity in [19], $\S 1$, and the fact that on a $d$-dimensional variety $V$ with only rational singularities any rational $d$-form which is regular on $V \backslash \operatorname{Sing} V$ remains regular on a resolution of singularities of $V$ (Kempf's criterion).

If further $\operatorname{dim} V \geq 3$ then outside a codimension 3 subvariety, $V$ is locally analytically a product of a germ of an RDP and a smooth germ. This uses repeated applications of Theorem 1.13 and the proof of Corollary 1.14 from [19].

We will use the following Lefschetz hyperplane section theorems.

Lemma 1. Let $(V, p)$ be a germ of an $n$-dimensional normal singular point of a complex analytic variety with $n>2$. Then we have the following assertions.

(i) For a general hyperplane section $L$ passing through $p$ it holds that the pair $(V \backslash \operatorname{Sing} V, L \backslash \operatorname{Sing} V)$ is $(n-2)$-connected.

(ii) For $c \in \mathbb{C}$ with $|c| \neq 0$ sufficiently small, the pair $(V \backslash \operatorname{Sing} V,\{L=c\} \backslash \operatorname{Sing} V)$ is $(n-1)$-connected ([11], Remark II.1.5).

Lemma 2. Let $V$ be a smooth quasi-projective variety of dimension $n$ embedded in $\mathbb{C}^{N}$. Then for a general hyperplane $L$ in $\mathbb{C}^{N}$, the pair $(V, V \cap L)$ is $(n-1)$-connected. ([10], Theorem 1.1.1). 
The next result was conjectured by P. Deligne and proved first in [7]), Part II, Theorem 1.1, and also later in [12], Theorem 3.1.1.

Lemma 3. Let $Z$ be a smooth quasi-projective irreducible variety of dimension $n$ with a dominant morphism $f: Z \rightarrow V$, where $V$ is an affine variety embedded as a closed subvariety in an affine space $\mathbb{C}^{N}$. Let L be a general linear subspace of $\mathbb{C}^{N}$ of codimension $c$. Then the pair $\left(Z,\left(f^{-1}(L)\right)\right.$ is $n_{1}$-connected, where

$$
n_{1}:=2 n-\sup [2 k+\sigma(k)+\inf (\sigma(k), c-1)]-1 .
$$

Here $\sigma(k)$ is the dimension of the set of points $p \in \mathbb{C}^{N}-L$ such that $\operatorname{dim} f^{-1}(p)$ is equal to $k$. (The dimension of an empty set is defined to be $-\infty$ ).

A similar statement is true for homology instead of homotopy groups.

Remark. With the notation of Lemma 3, if the set where $\operatorname{dim} f^{-1}(p)=k$ is empty then $\sigma(k)=-\infty$ and we need not consider such terms while calculating the supremum.

The theory of tubular neighborhoods is a well-developed theory in Stratified Morse Theory ([7], Part II, Chapter 2). In our proof we need to take tubular neighborhoods of singular subvarieties of singular algebraic varieties. Because of the singularities of the subvariety (or because of "non-equisingular" points of the subvariety) the arguments are somewhat technical but the underlying idea is not difficult. The proof of the theorem is less technical if the singular locus of $W$ is finite.

\section{Proof of the theorem}

We use the notation from the statement of the theorem from the introduction.

If $\mathbb{C}^{n} / / G^{0}$ is a surface then it is proved in [9] that $\mathbb{C}^{n} / / G^{0} \cong \mathbb{C}^{2}$. From this, using the argument given at the beginning of the proof of (a) below we deduce easily that the theorem is true if $\operatorname{dim} W=2$. Hence theorem is proved if $\operatorname{dim} W=2$. From now onwards we will assume that $\operatorname{dim} W \geq 3$.

If $G$ is connected and semisimple then it is well known that the coordinate ring $R$ of $W$ is a UFD and for any subvariety $S$ of codimension $\geq 2$ in $W$ its inverse image $\pi^{-1}(S)$ has codimension $\geq 2$ in $\mathbb{C}^{n}$ ([13]). Since we want to use this result in a more general situation later on we will indicate the argument briefly. Let $D$ be an irreducible divisor in $W$. Then $\pi^{*}(D)=(r)$ for some regular function on $\mathbb{C}^{n}$. Then $r$ is a semi-invariant. Hence for the action of $G$ we have $\rho_{g}(r)=u_{g} \cdot r$, where $u_{g}$ is a unit in the coordinate ring of $\mathbb{C}^{n}$. This implies that $u_{g}$ is a non-zero constant. Now $u_{g}$ gives a character on $G$. Since $G$ is connected and semisimple $u_{g}$ is a constant which is independent of $g$. Hence $u_{g} \equiv 1$. Thus $r$ is an invariant regular function 
Vol. 84 (2009) Topological properties of quotients modulo semisimple algebraic groups 797

and $(D)=(r)$. By the same argument we show that if $S \subset W$ is an irreducible subvariety of codimension $\geq 2$ then $\pi^{-1} S$ has codimension $\geq 2$ in $\mathbb{C}^{n}$.

$W$ has rational singular points ([2]) and it is contractible (for this latter property it suffices for $G$ to be reductive, see [14]). We will use these results in what follows.

It will be clear from the proof of the theorem that we will only use $\pi_{1}\left(\mathbb{C}^{n}\right)=$ (1), $H_{i}\left(\mathbb{C}^{n}\right)=(0)$ for $1 \leq i \leq 3$. This remark will be implicitly used later in more general situations.

Proof of (a). As remarked in the introduction, it is proved in [8] that the group $\pi_{1}^{p}(W)$ is finite if $G$ is reductive. Hence $H_{1}^{p}(W)$ is also finite.

For the proof of part (b) below we need only reductivity of $G$. Suppose that $G^{0}$ is semisimple. Then we have a factorization $\mathbb{C}^{n} \rightarrow \mathbb{C}^{n} / / G^{0} \rightarrow \mathbb{C}^{n} / / G$. If $H_{2}^{p}\left(\mathbb{C}^{n} / / G^{0}\right)$ is finite for any point $p \in \mathbb{C}^{n} / / G^{0}$ then this is also true for $\mathbb{C}^{n} / / G$. For this we use the fact that if $Z \rightarrow Y$ is a proper morphism with finite fibers from a normal complex space $Z$ onto a normal complex space $Y$ then the induced homomorphism $H_{i}(Z) \rightarrow H_{i}(Y)$ has image of finite index for $i \geq 0$. This can be proved using triangulations of $Y, Z$ (or using the transfer map, [6]).

In view of these observations for the rest of the proof of the theorem, except in (b), we will assume that $G$ is connected and semisimple.

First we will prove that for any point $p \in W$ the local first homology group $H_{1}^{p}(W)=(0)$.

This is obvious if $p$ is a smooth point of $W$ since $\operatorname{dim} W>1$ so we will assume that $p \in \operatorname{Sing} W$. Let $S:=\operatorname{Sing} W$.

We can find a suitable contractible open neighborhood $N$ of $p$ in $W$, a slightly bigger contractible open neighborhood $N^{\prime}$ of $p$ such that the closure $\bar{N} \subset N^{\prime}$ and we use the covering $W \backslash(S \cap \bar{N}), N^{\prime}$ of $W$ by open subsets of $W$ and the corresponding Mayer-Vietoris sequence for homology

$$
H_{2}(W) \rightarrow H_{1}\left(N^{\prime} \backslash(S \cap \bar{N})\right) \rightarrow H_{1}(W \backslash(S \cap \bar{N})) \oplus H_{1}\left(N^{\prime}\right) \rightarrow H_{1}(W) \rightarrow \cdots
$$

Since $W, N, N^{\prime}$ are contractible we have $H_{1}(W)=(0)=H_{2}(W)=H_{1}\left(N^{\prime}\right)$. Hence we get $H_{1}\left(N^{\prime} \backslash(S \cap \bar{N})\right) \cong H_{1}(W \backslash(S \cap \bar{N}))$. Since $\pi^{-1}(S)$ has codimension $\geq 2$ we also have $\pi_{1}\left(\mathbb{C}^{n} \backslash \pi^{-1}(S)\right)=(1)$ and using the fact that $\pi$ has connected fibers we get $\pi_{1}(W \backslash S)=(1)$. Now $S$ is a proper simplicial subcomplex of $W$ of real codimension $\geq 4$ and since $W$ is normal $S$ does not disconnect $W$ at any point. Hence any 1-cycle in $W \backslash(S \cap \bar{N})$ can be deformed to a 1-cycle lying in $W \backslash S$. Therefore the map $H_{1}(W \backslash S) \rightarrow H_{1}(W \backslash(S \cap \bar{N}))$ is onto. Hence $H_{1}(W \backslash(S \cap \bar{N}))=(0)$. Thus, $H_{1}\left(N^{\prime} \backslash(S \cap \bar{N})\right)=(0)$. Finally, using the same argument as above we see that the map $H_{1}\left(N^{\prime} \backslash(S \cap \bar{N})\right) \rightarrow H_{1}\left(N^{\prime} \backslash S\right)$ is surjective. Hence $H_{1}\left(N^{\prime} \backslash S\right) \cong H_{1}^{p}(W)=(0)$.

Proof of (b). For this part we only need reductivity of $G$. 
Now assume that $W$ has isolated singularities. Let $W \subset X$ be an embedding into a normal projective variety $X$ such that $X$ is smooth outside $W$ and $X \backslash W$ is a simple normal crossing divisor $D$. Let $N$ be a suitable tubular neighborhood of $D$ in $X$. Then $\pi_{1}(N \backslash D)$ is the fundamental group at infinity of $W$. A Lefschetz-type result of Hamm- Lê Dũng Tráng says that $X \backslash(S \cup D)=W \backslash S$ has the homotopy of a space obtained from $N \backslash D$ by attaching cells of dimension $\geq 3$ ([10]). Since $\pi_{1}(W \backslash S)=(1)$, we conclude that $\pi_{1}(N \backslash D)=(1)$.

Proof of (c). The proof of this main part of the theorem is rather involved.

First we will prove that $H_{2}(W \backslash S)$ is finite. This result and its proof plays a crucial role in the proof of finiteness of $H_{2}^{p}(W)$ at any point.

Since $H_{2}\left(\mathbb{C}^{n} \backslash \pi^{-1} S\right)=(0)$ it suffices to show that the map $H_{2}\left(\mathbb{C}^{n} \backslash \pi^{-1} S\right) \rightarrow$ $H_{2}(W \backslash S)$ has image of finite index. Let $U \subset W \backslash S$ be the principal Luna stratum for $\pi$ ([16], Chapter III, §3). $U$ is a non-empty Zariski-open subset of $W \backslash S$ such that $\pi: \pi^{-1}(U) \rightarrow U$ is a locally analytically trivial fiber bundle.

Case 1. $W \backslash U$ does not contain any divisor.

In this case codimension $W \backslash U \geq 2$. It is shown in the proof of part (a) that $H_{1}(W \backslash S)=(0)$. From these two it follows that $\pi_{1}(U)=\pi_{1}\left(\pi^{-1} U\right)=$ $(1), \pi_{2}\left(\pi^{-1} U\right)=(0)$. By Hurewicz's theorem $H_{2}\left(\pi^{-1} U\right)=(0)$. Let $F$ be a general fiber of $\pi$. It is known that $F$ has the homotopy type of a closed fiber $G / H$, where $H$ is a closed reductive subgroup of $G$ ([16], III, §3]). Consider the long exact homotopy sequence

$$
\pi_{2}(F) \rightarrow \pi_{2}\left(\pi^{-1} U\right) \rightarrow \pi_{2}(U) \rightarrow \pi_{1}(F) \rightarrow \cdots .
$$

For $\pi_{1}(F)$ we have the exact sequence

$$
\pi_{1}(H) \rightarrow \pi_{1}(G) \rightarrow \pi_{1}(G / H) \rightarrow \pi_{0}(H) .
$$

Since $G$ is semisimple, $\pi_{1}(G)$ is finite and $\pi_{0}(H)$ has the cardinality of the set of connected components of $H$. Thus $\pi_{1}(G / H)$ is finite. This implies that $\pi_{1}(F)$ is finite. It follows that $\pi_{2}(U)$ is finite. Since $\pi_{1}(U)=(1)=\pi_{1}\left(\pi^{-1} U\right)$, by the Generalized Whitehead Theorem on the relation between homotopy and homology ([20], [Chapter 9, §6 ]) the map $H_{2}\left(\pi^{-1} U\right) \rightarrow H_{2}(U)$ has image of finite index. So we get that $H_{2}(U)$ is finite. Since $W \backslash S$ is smooth and $(W \backslash S) \backslash U$ has no divisors, $H_{2}(U) \cong H_{2}(W \backslash S)$, proving that $H_{2}(W \backslash S)$ is finite.

Case 2. $W \backslash U$ has divisors.

We will let $U$ be an affine open subset of $W$ such that $U$ is contained in the principal stratum for $\pi$. Let $D:=W \backslash U$. Let $D=\cup D_{i}$ be the decomposition of $D$ into irreducible components $D_{i}$. Since the coordinate ring of $W$ is a UFD each $D_{i}$ is a principal divisor, say $D_{i}=\left(f_{i}\right)$. 
Vol. 84 (2009) Topological properties of quotients modulo semisimple algebraic groups 799

Now let $L$ be a general hyperplane in the affine space in which $W$ is embedded as a closed subvariety and let $Y:=W \cap L$. By Lemma $2, \pi_{i}(W \backslash S, Y \backslash S)=(0)$ for $i \leq d-1$, where $d=\operatorname{dim} W$. In particular, $\pi_{2}(Y \backslash S) \rightarrow \pi_{2}(W \backslash S)$ is onto and $\pi_{1}(Y \backslash S) \cong \pi_{1}(W \backslash S)=(1)$. This implies that the map $H_{2}(Y \backslash S) \rightarrow H_{2}(W \backslash S)$ is onto, again by Whitehead's theorem. For general $L, X:=\pi^{-1}(Y)$ is smooth and irreducible by Bertini theorems. $X$ is $G$-stable and $Y=X / / G$.

Since $\pi^{-1} S$ has codimension $\geq 2$ in $\mathbb{C}^{n}$, we have $H_{2}\left(\mathbb{C}^{n} \backslash \pi^{-1} S\right)=(0)$. Since the compositions of the morphisms $X \rightarrow \mathbb{C}^{n} \rightarrow W$ and $X \rightarrow Y \rightarrow W$ are same the compositions of the induced maps on homology groups are the same. Therefore it suffices to show that the map $\left.H_{2}\left(X \backslash \pi^{-1} S\right)\right) \rightarrow H_{2}(Y \backslash S)$ has image of finite index.

In order to illustrate the crucial idea we will assume that $\operatorname{dim} W=3$. The general case is proved exactly similarly by taking general hyperplane sections of $W$ till we get a 3-dimensional variety. Since $X$ is smooth, by Boutot's result $Y$ also has rational singularities. Since $W$ is Cohen-Macaulay and $\Gamma(W, \mathcal{O})$ is a $\mathrm{UFD}, W$ is also Gorenstein. It follows that $Y$ is a Gorenstein surface with rational singularities.

We claim that $Y$ has at most $E_{8}$-singularities.

By part (a), the group $H_{1}^{p}(W)=(0)$. If $W$ has only isolated singularities then $Y$ is smooth. So we can assume that Sing $W$ contains a curve. It is well known that RDPs have no moduli ([1], §1). Hence at a point in $\operatorname{Sing} Y, W$ is locally analytically a product of the germ $(Y, p)$ and a disc in $\mathbb{C}$. It follows from $H_{1}^{p}(W)=(0)$ that $H_{1}^{p}(Y)=(0)$. The only RDP which has trivial local $H_{1}$ is $E_{8}$.

Thus $Y$ has only $E_{8}$ singularities. Hence $Y$ is a topological manifold at each of its points. For general $L$, the divisor $D_{i} \cap L$ is irreducible and defined by $\left.f_{i}\right|_{Y}$.

Claim. The homomorphism $\mathrm{H}_{2}(U \cap L) \rightarrow \mathrm{H}_{2}(Y)$ has image of finite index.

This will follow by induction on the number of irreducible components of $D$ from the following result. Using the observation that $Y$ is a topological manifold, for the purpose of illustrating the crucial idea we can assume that $Y$ is smooth.

Lemma 4. Let $Z$ be a smooth affine surface and let $C \subset Z$ an irreducible curve which is a principal divisor $(f)$. Then the homomorphism $\mathrm{H}_{2}(Z \backslash C) \rightarrow \mathrm{H}_{2}(Z)$ is a surjection.

Proof. First we will give the proof when $C$ is smooth. Let $N$ be a suitable tubular neighborhood of $C$ in $Z$. Since $Z, C$ are affine $N$ is a topologically trivial 2-disc bundle over $C$ and hence its boundary is a trivial $S^{1}$-bundle over $C$. We use the open cover $Z \backslash C, N$ of $Z$ and consider the corresponding Mayer-Vietoris sequence

$\rightarrow H_{2}(\partial N) \rightarrow H_{2}(Z \backslash C) \oplus H_{2}(N) \rightarrow H_{2}(Z) \rightarrow H_{1}(\partial N) \rightarrow H_{1}(Z \backslash C) \oplus H_{1}(N)$.

We will prove that the map $H_{1}(\partial N) \rightarrow H_{1}(Z \backslash C) \oplus H_{1}(N)$ is injective. Now $\partial N=C \times S^{1}$ and $C$ is a strong deformation retract of $N$. The function $f$ gives a 
morphism $Z \backslash C \rightarrow \mathbb{C}^{*}$ such that the image of a small loop $\gamma$ around $C$ in $Z$ maps onto the generator of $H_{1}\left(\mathbb{C}^{*}\right)$. Hence $\gamma$ has infinite order in $H_{1}(Z \backslash C)$. From this injectivity of the map $H_{1}(\partial N) \rightarrow H_{1}(Z \backslash C) \oplus H_{1}(N)$ is easy to deduce.

Again, since $C$ is a strong deformation retract of $N$ and $C$ is an affine curve we see that $H_{2}(N)=(0)$. Hence $H_{2}(Z \backslash C) \rightarrow H_{2}(Z)$ is surjective.

This proves the result when $C$ is smooth. If $C$ has (finitely many) singular points then we work with $C \backslash \operatorname{Sing} C$ and $Z \backslash \operatorname{Sing} C$. Now $Z \backslash \operatorname{Sing} C$ is not affine but $C \backslash \operatorname{Sing} C$ is affine. Also, $H_{2}(Z \backslash \operatorname{Sing} C) \cong H_{2}(Z)$ since Sing $C$ is a finite set. Since $C \backslash \operatorname{Sing} C$ is affine $H_{2}(C \backslash \operatorname{Sing} C)=(0)$. This is enough for the above proof.

Remark. The assumption that $C$ is a set-theoretically principal divisor is necessary. As an example we consider the affine quadric $Q:=\left\{x^{2}+y^{2}+z^{2}=1\right\} \subset \mathbb{C}^{3}$. It contains $\mathbb{C}^{2}$ as an affine open subset such that $Q \backslash \mathbb{C}^{2} \cong \mathbf{A}^{1}$. But $H_{2}(Q) \cong \mathbf{Z}$ and $\mathrm{H}_{2}\left(\mathbb{C}^{2}\right)=(0)$. It is easy to see that $Q \backslash \mathbb{C}^{2}$ generates the infinite cyclic divisor class group of $Q$.

Now $U \cap L \subset Y$ is contained in the principal stratum for $\left.\pi\right|_{X}$. Hence all the fibers of $X \rightarrow Y$ for points in $U \cap L$ are mutually isomorphic. We can now use the following result which follows from a general result due to Kraft-Russell ([15], §3, Proposition).

Lemma 5. There is an étale dominant morphism $h: V \rightarrow U$ such that the induced map $V \times_{U} \pi^{-1}(U) \rightarrow V$ is a trivial bundle $V \times F$, where $F$ is a general fiber of $\pi$.

Using the fact that $\Gamma(W, \mathcal{O})$ is a UFD we can now find an affine open subset $U_{0} \subset U \cap L$ such that the complement $(U \cap L) \backslash U_{0}$ is a union of irreducible curves $\Delta_{i}$ each of which is a principal divisor in $Y$. Further, we can assume that the map $h: V_{0}:=h^{-1}\left(U_{0}\right) \rightarrow U_{0}$ is a finite morphism. By Lemma 4 we know that the map $H_{2}\left(U_{0}\right) \rightarrow H_{2}(Y)$ is onto. Clearly the map $H_{2}\left(V_{0} \times F\right) \rightarrow H_{2}\left(V_{0}\right)$ is onto. Since the map $V_{0} \rightarrow U_{0}$ is finite the induced map $H_{2}\left(V_{0}\right) \rightarrow H_{2}\left(U_{0}\right)$ has image of finite index. This follows from the observation that $V_{0}, U_{0}$ can be triangulated so that the map $V_{0} \rightarrow U_{0}$ is simplicial ([6]). Alternatively, we can use the Transfer map in the theory of finite group actions on simplicial complexes. It follows now that the map $H_{2}\left(X \backslash \pi^{-1} S\right) \rightarrow H_{2}(Y \backslash S)$ has image of finite index.

We use this to prove (c) first in the case when $W$ has isolated singularities.

Let $p_{1}, p_{2}, \ldots, p_{r}$ be all the singular points of $W$ and let $N_{i}$ be a small contractible neighborhood of $p_{i}$ in $W$. We use the covering $W \backslash S, \cup N_{i}$ of $W$ and the corresponding Mayer-Vietoris sequence

$$
H_{3}(W) \rightarrow H_{2}\left(\cup\left(N_{i} \backslash p_{i}\right)\right) \rightarrow H_{2}(W \backslash S) \oplus H_{2}\left(\cup N_{i}\right) \rightarrow H_{2}(W) \rightarrow \cdots .
$$


Since $W, N_{i}$ are contractible and $H_{2}(W \backslash S)$ is finite as proved above we deduce that $H_{2}\left(N_{i} \backslash p_{i}\right)$ is finite for $i=1,2, \ldots, r$. This means the local second homology groups $H_{2}^{p_{i}}(W)$ are all finite. This proves (c) when $W$ has isolated singular points.

For the general case, again first we give the $\operatorname{argument}$ when $\operatorname{dim} W=3$.

Now the singular locus of $W$ can be assumed to be a disjoint union of a curve $C$ and a finite set of points, say $\left\{q_{1}, q_{2}, \ldots, q_{s}\right\}$. We have shown earlier that a general hyperplane section $W \cap L$ is Gorenstein and has only $E_{8}$ singularities. Clearly, a general hyperplane does not pass through any of the $q_{j}$. Similarly, there is a finite set $\left\{p_{1}, p_{2}, \ldots, p_{r}\right\} \subset C$ such that a general hyperplane section $W \cap L$ not passing through any $p_{i}$ has at worst $E_{8}$ as singularities (here, we are using Lemma 1 (ii) and part (a) of the theorem which is already proved). It is well known that a flat deformation of an RDP is locally trivial outside the special fiber. Hence by removing finitely many more points in $C$, if necessary, we deduce that the variety $W$ is locally analytically a product of a 1-dimensional disc in $\mathbb{C}$ and the germ of the $E_{8}$ singularity for any $p \in C \backslash\left\{p_{1}, \ldots, p_{r}\right\}$.

Let $P:=\left\{p_{1}, \ldots, p_{r}, q_{1}, \ldots, q_{s}\right\}$. At each point in $W \backslash P, W$ is a topological manifold. The previous proof works without any change to show that $H_{2}(W \backslash P)$ is finite. Using the open cover $W \backslash P, \cup N_{i}$, where $N_{i}$ are suitable contractible neighborhoods of the points in $P$, and the Mayer-Vietoris sequence we deduce that $H_{2}^{q_{j}}(W)$ is finite for any $q_{j}$.

Remark. The proof also shows that $H_{2}\left(N_{i}-p_{i}\right)$ is finite. But we have $H_{2}^{p_{i}}(W)=$ $H_{2}\left(N_{i} \backslash C\right)$ so we cannot conclude immediately that $H_{2}^{p_{i}}(W)=(0)$.

Let $N$ be a suitable tubular neighborhood of $C$ in $W$. Let $S_{i}$ be a general hyperplane section of $W$ at $p_{i}$ for $1 \leq i \leq r$. Since $W$ is 3-dimensional, normal and Cohen-Macaulay, $S_{i}$ is analytically irreducible at $p_{i}$ by Flenner's local Bertini theorem ([3]). Now $N \backslash \cup_{i} S_{i}$ is a tubular neighborhood of $C \backslash\left\{p_{1}, \ldots, p_{r}\right\}$ in $W \backslash \cup S_{i}$.

Claim. $\mathrm{H}_{2}(\mathrm{~N} \backslash \mathrm{C})$ is finite.

For proving this we consider the cover $W \backslash C, N$ of $W$. Using the Mayer-Vietoris sequence for this cover and the result that $\mathrm{H}_{2}(W \backslash S)=(0)$ proved above proves the claim.

Now we can write $N=U_{1} \cup U_{2}$, where $U_{1}$ is a disjoint union of neighborhoods of $p_{1}, \ldots, p_{r}$ in $N$ and $U_{2}$ is a bundle over $C \backslash \Delta$, where $\Delta$ is a disjoint union of small neighborhoods of $p_{1}, \ldots, p_{r}$ in $C$, whose fiber is a germ of an $E_{8}$ singularity. Then $U_{1} \backslash C, U_{2} \backslash C$ is an open cover of $N \backslash C$. The intersection $\left(U_{1} \backslash C\right) \cap\left(U_{2} \backslash C\right)$ has the homotopy type of a bundle over $\partial \Delta$ (which is a disjoint union of finitely many circles) whose fiber has the homotopy type of the link of $E_{8}$ singularity. Now the link of the $E_{8}$ singularity is a quotient of the 3 -sphere $S^{3}$ by the binary icosahedral group $P$ of 
order 120. We have an exact sequence (1) $\rightarrow P \rightarrow \pi_{1}(E) \rightarrow \pi_{1}\left(S^{1}\right) \rightarrow(1)$, where $S^{1}$ is one of the connected components of $\partial \Delta$ and $E$ is the total space of the bundle over this $S^{1}$. This sequence splits since $\pi_{1}\left(S^{1}\right) \cong \mathbf{Z}$. Write $\pi_{1}(E)=P \cdot(\gamma)$, where $P$ is normal in $\pi_{1}(E)$ and $(\gamma)$ maps isomorphically onto $\mathbf{Z}$ in the above short exact sequence. Since $(\gamma)$ has finite index (equal to 120 ) in $\pi_{1}(E)$, we can find a subgroup of finite index, say $\left(\gamma^{\prime}\right) \subset(\gamma)$ such that $\left(\gamma^{\prime}\right)$ is normal in $\pi_{1}(E)$. This implies that there is a finite normal covering $E^{\prime} \rightarrow E$ whose fundamental group is the normal subgroup $P \cdot\left(\gamma^{\prime}\right)$ of $\pi_{1}(E)$. There is a fiber bundle $E^{\prime} \rightarrow S^{1}$ with fiber the link of the $E_{8}$ singular point. Further, since $P \cdot\left(\gamma^{\prime}\right)$ is normal in $\pi_{1}(E)$ by taking the universal covers of the fibers of the bundle $E^{\prime} \rightarrow S^{1}$ we get an $S^{3}$-bundle over $S^{1}$. It follows that there is an orientable $S^{3}$-bundle over $\partial \Delta$ such that $\left(U_{1} \backslash C\right) \cap\left(U_{2} \backslash C\right)$ has the homotopy type of the quotient of this $S^{3}$-bundle over $\partial \Delta$. Since an orientable sphere bundle over $S^{1}$ is trivial we see that the total space of this $S^{3}$-bundle has trivial second homology and hence $H_{2}\left(\left(U_{1} \backslash C\right) \cap\left(U_{2} \backslash C\right)\right)$ is also finite. Using the fact that $\pi_{1}(C \backslash \Delta)$ is a free group we observe similarly that the space $U_{2} \backslash C$ is a quotient of an orientable $S^{3}$-bundle $E^{\prime} \rightarrow C^{\prime}$, where $C^{\prime}$ is a finite-sheeted covering space $C^{\prime} \rightarrow C \backslash \Delta$. Using the Gysin sequence of an $S^{3}$-bundle over $C^{\prime}$ we first see that $H_{2}\left(E^{\prime}\right)$ is finite and hence $H_{2}\left(U_{2} \backslash C\right)$ is also finite. Now we use the Mayer-Vietoris sequence for the covering $U_{1} \backslash C, U_{2} \backslash C$ of $N \backslash C$ and the result that $H_{2}(N \backslash C)$ is finite as proved above to conclude that $H_{2}\left(U_{1} \backslash C\right)$ is finite. This proves that the local second homology group of each $p_{i}$ is finite.

This completes the proof of part (c) of the theorem when $\operatorname{dim} W=3$.

Next we indicate the modification of the above proof when $\operatorname{dim} W=4$.

Case 1. Assume that $W$ has only isolated singularities.

We have already shown that $H_{2}(W \backslash S)$ is finite.

Since $W$ has isolated singularities, as in the case $\operatorname{dim} W=3$ we prove that the local second homology group at any point of $W$ is finite.

Case 2. Assume that Sing $W$ is a disjoint union of a curve $C$ and finitely many points $\left\{p_{i}\right\}$.

Let $N$ be a suitable tubular neighborhood of $C$ and let $N_{i}$ be suitable contractible neighborhoods of $p_{i}$. Consider the open cover $X \backslash \operatorname{Sing} W, N \cup\left(\cup_{i} N_{i}\right)$ of $W$ and the corresponding Mayer-Vietoris sequence. Using the finiteness of $H_{2}(W \backslash \operatorname{Sing} W)$ proved earlier we conclude that $H_{2}(N \backslash C), H_{2}^{p_{i}}(W)$ are all finite.

Let $Y$ be a general hyperplane section of $W$ and let $X:=\pi^{-1}(Y)$. Then $X$ is smooth, $G$ stable and $Y=X / / G$. We will apply Lemma 3 to the map $\pi: \mathbb{C}^{n} \rightarrow W$ with $c=1$.

The integer $n_{1}=2 n-1-\sup _{k}[2 k+\sigma(k)]$, where the supremum is taken over those $k$ such that there is at least one fiber of $\pi$ of dimension $k$. We claim that $n_{1} \geq 3$. This is equivalent to $2 n-4 \geq \sup _{k}[2 k+\sigma(k)]$. We have observed in the beginning of the proof of the theorem that the inverse image of any subvariety of $W$ of codimension 
Vol. 84 (2009) Topological properties of quotients modulo semisimple algebraic groups 803

$\geq 2$ has codimension $\geq 2$ in $\mathbb{C}^{n}$. Since $\operatorname{dim} W \geq 4$, any fiber of $\pi$ has codimension $\geq 2$. This implies that $k+\sigma(k) \leq n-2$, so that $2 k+2 \sigma(k) \leq 2 n-4$.

Now by Lemma $3, H_{i}(X)=(0)$ for $i=1,2$. Similarly, $\pi_{1}(X)=(1)$ so that a smooth compactification of $X$ is also simply-connected and $X$ does not have nonconstant regular invertible functions. Now for any smooth compactification $\bar{X}$ of $X$ we have $H_{1}(\bar{X})=(0)$, so that $P i c_{0}(\bar{X})=0$. From $H_{1}(X)=(0)=H_{2}(X)$ we observe that $H^{2}(X)=(0)$. The group $\hat{\mathrm{H}}^{2}(X)$ defined in [5], $\S 1$, is a subgroup of $H^{2}(X)$, which in our situation is trivial. Now it follows that the coordinate ring of $X$ is a UFD ([5], Corollary 1.20). Since $G$ is connected and semisimple, as seen in the beginning, the coordinate ring of $Y$ is also a UFD. Now $Y$ has isolated singularities. Let $p \in \operatorname{Sing} Y$. By Lemma 1 the homomorphism $H_{2}^{p}(Y) \rightarrow H_{2}^{p}(W)$ is an isomorphism. It suffices to show that $H_{2}^{p}(Y)$ is finite. The inverse image in $X$ of any subvariety of codimension $\geq 2$ of $Y$ has codimension $\geq 2$ in $X$. From this we see that there are only finitely many points in $Y$ whose inverse image has codimension 2 in $X$.

Let $Z$ be a general linear space section of $X$ of dimension 3. By Bertini's theorem $Z$ is smooth and irreducible. By abuse of notation we denote $\pi: Z \rightarrow Y$ to be the restriction morphism. Now $\pi$ is a generically finite morphism and there are only finitely many points, say $p_{1}, p_{2}, \ldots, p_{r}$, in $Y$ whose inverse image in $Z$ is codimension 2 (the intersection of $Z$ with some codimension 2 subvarieties of $X$ ), i.e. it is 1-dimensional. Let $p=p_{1}$. If $\pi^{-1}(p)$ is finite then $Y$ has a quotient singularity at $p$ and hence $H_{2}^{p}(Y)$ is finite. So let $D:=\pi^{-1}(p)$. Then $D$ is a (possibly reducible) curve. Since $\pi$ is generically finite we infer that there are finitely many irreducible divisors $\Delta_{1}, \ldots, \Delta_{l}$ in $Y$ such that $\pi$ restricts to a proper morphism with finite fibers $Z \backslash \pi^{-1}\left(\cup \Delta_{i}\right) \rightarrow Y \backslash\left(\cup \Delta_{i}\right)$. Since we are only interested in the topology of a small neighborhood of $p$ we will assume, for simplicity, that $p$ is the only singular point of $Y$ and $p \in \Delta_{i}$ for each $i$.

Let $U$ be a suitable Stein contractible neighborhood of $p$ in $Y$. Each $\Delta_{i}$ is a principal divisor in $Y$ since the coordinate ring of $Y$ is a UFD.

We use Lemma 1. If $H$ is a general hyperplane section of $U$, not passing through $p$, then the natural map $H_{2}(H) \rightarrow H_{2}(U \backslash\{p\})$ is a surjection. $H$ is a 2-dimensional Stein manifold. Since each $\Delta_{i}$ is a principal divisor, by the proof of Lemma 4 we see that the map $H_{2}\left(H \backslash \cup \Delta_{i}\right) \rightarrow H_{2}(H)$ is a surjection. Hence the composite map $H_{2}\left(H \backslash \cup \Delta_{i}\right) \rightarrow H_{2}(U \backslash\{p\})$ is also a surjection. Let $T$ be a suitable tubular neighborhood of $D$ in $Z$ such that $D$ is a strong deformation retract of $T$ and $\pi(T) \subset$ $U$. It follows that $H_{2}(T)=(0)$. Let $U_{1} \subset U$ be a slightly smaller neighborhood of $p$ which is a strong deformation retract of $U$, so that $H_{2}\left(U_{1} \backslash\{p\}\right) \cong H_{2}(U \backslash\{p\})$. By shrinking $U_{1}$ we can assume that the inverse image $\pi^{-1}\left(U_{1}\right)$ is contained in $T$. The map $H_{2}\left(\pi^{-1}\left(\left(H \cap U_{1}\right) \backslash \cup \Delta_{i}\right)\right) \rightarrow H_{2}\left(\left(H \cap U_{1}\right) \backslash \cup \Delta_{i}\right)$ has image of finite index since the morphism $\pi^{-1}\left(\left(H \cap U_{1}\right) \backslash \cup \Delta_{i}\right) \rightarrow\left(H \cap U_{1}\right) \backslash \cup \Delta_{i}$ is proper with finite fibers. Hence the composite map $H_{2}\left(\pi^{-1}\left(\left(H \cap U_{1}\right) \backslash \cup \Delta_{i}\right)\right) \rightarrow H_{2}(U \backslash\{p\})$ 
also has image of finite index. Now $H_{2}(T \backslash D)=(0)$ since $D$ is an affine curve and has codimension $\geq 2$ in $T$. The map $H_{2}\left(\pi^{-1}\left(\left(H \cap U_{1}\right) \backslash \cup \Delta_{i}\right)\right) \rightarrow H_{2}(U \backslash\{p\})$ factors through $H_{2}(T \backslash D)$, which is trivial. Hence we conclude that $H_{2}(U \backslash\{p\})$ is finite.

This completes the proof of Case 2.

Case 3. Assume finally that $\operatorname{Sing} W$ contains a surface $S$.

A general hyperplane section $W \cap L_{1}$ is Gorenstein of dimension 3 and has rational singularities. Its singular locus is $\operatorname{Sing} W \cap L_{1}$. In the terminology of [19], $W \cap L_{1}$ is a Gorenstein canonical singularity. Another general hyperplane section gives $W \cap L_{1} \cap L_{2}$, which is also Gorenstein and has rational singularities. As seen before, the singularities of $W \cap L_{1} \cap L_{2}$ are $E_{8}$-singularities. At any such point $W \cap L_{1} \cap L_{2}$ is topologically a manifold. Using the observation that RDPs have no moduli, it is shown in [19], Corollary 1.14, that outside a codimension 3 subvariety, say $F, W$ is locally analytically a product of an RDP and the unit disc in $\mathbb{C}^{2}$. Now $F$ is a disjoint union of a curve $C \subset W$ and a finite set of points such that any point $p \notin F$ is either a smooth point of $W$ or $W$ is locally analytically a product (disc in $\mathbb{C}^{2}$ ) $\times$ germ of $E_{8}$. Hence $H_{2}^{p}(W)$ is trivial for any such point $p$. Clearly $W$ is a topological manifold at any point in $W \backslash F$. We prove that $H_{2}^{p}(W)$ is finite for any $p \in C$ exactly by the same argument as in Case 2 .

This completes the proof of assertion (c) of the theorem when $\operatorname{dim} W=4$.

Consider the case when $\operatorname{dim} W>4$. We will indicate the proof when $\operatorname{dim} W=5$ and leave the general case to the reader.

Arguing as in the case $\operatorname{dim} W=4$ above, we see that there is a codimension 3 subvariety, say $F$, of $W$ such that $W$ is at any point $p \in W \backslash F$ locally analytically a product (disc in $\mathbb{C}^{3}$ ) $\times$ germ of $E_{8}$ singularity. In particular, $W$ is a topological manifold at any point in $W \backslash F$. Now $F$ is a union of a surface $S$, a curve $C$ and finitely many points not contained in $S \cup C$. We prove that $H_{2}(W \backslash F)$ is finite as before. We ignore the finitely many isolated points in $F$. Next, if $N$ is a suitable tubular neighborhood of $F$ in $W$ then using a Mayer-Vietoris sequence for the cover $(W \backslash F), N$ we deduce that the natural map $H_{2}(N \backslash F) \rightarrow H_{2}(N)$ is an isomorphism.

Let $Y:=W \cap L$, where $L$ is a general linear subspace of $\mathbb{C}^{N}$ of codimension 2. Let $X:=\pi^{-1}(Y)$. Now $\operatorname{dim} Y=3$ and $Y \cap F$ is a finite set of points. If we apply Lemma 3 with $c=2$ then we see as in the proof of Case 2 (when $\operatorname{dim} W=4$ ) that the pair $\left(\mathbb{C}^{n}, X\right)$ is 3-connected. Then $H_{i}(X)=(0)$ for $i=1,2$, the coordinate ring of $X$ is a UFD and has no regular invertible functions. This implies as in the proof of Case $2(\operatorname{dim} W=4)$ that the coordinate ring of $Y$ is a UFD, the inverse image of any codimension $\geq 2$ subvariety of $Y$ has codimension $\geq 2$ in $X$, etc. We prove that $H_{2}^{p}(Y)$ is finite exactly as in the proof of Case $2(\operatorname{dim} W=4)$. Finally, using Lemma 1 we deduce that $H_{2}^{p}(W)$ is finite.

This completes the proof of the theorem. 
Vol. 84 (2009) Topological properties of quotients modulo semisimple algebraic groups 805

Remark. T. N. Venkataramana has shown us an interesting construction of the following phenomenon.

Let $\Gamma$ be a non-trivial finite perfect group. Then there is a suitable linear action of $G=S L\left(N, \mathbb{C}\right.$ ) (for $N>>0$ ) on $\mathbb{C}^{N}$ such that for a suitable point $p$ in $\mathbb{C}^{N} / / G$ the local fundamental group of $\mathbb{C}^{N} / / G$ at $p$ is $\Gamma$. On the other hand, it is easy to see from our proof that in assertion (a) of the theorem the local fundamental group at the vertex in $\mathbb{C}^{N} / / G$ is trivial.

\section{Proof of the corollary}

Let $G, W=\mathbb{C}^{n} / / G, p \in W$ be as in the statement of the corollary of the theorem as stated in the Introduction. As remarked in the beginning $W$ has rational singularities. For rational singularities (in arbitrary dimension) Flenner has shown ([4], §6, Satz 6.1) that the analytic local ring $\mathcal{O}_{W, p_{\text {an }}}$ is a UFD if and only if there exists a fundamental system of Stein contractible neighborhoods $U$ of $p$ in $W$ such that $H^{2}(U \backslash \operatorname{Sing} W)=(0)$. This is equivalent to the two conditions $H_{1}(U \backslash$ Sing $W)$ is torsion-free and $H_{2}(U \backslash$ Sing $W)$ is finite. By the parts (a) and (c) of the theorem these are both true for $W$.

An example. Let $\mathbb{C}^{*}$ act on $\mathbb{C}^{4}$ by $\rho_{t}\left(X_{1}, X_{2}, X_{3}, X_{4}\right)=\left(t X_{1}, t X_{2}, t^{-1} X_{3}, t^{-1} X_{4}\right)$. The ring of invariants is $\mathbb{C}\left[X_{1} X_{3}, X_{1} X_{4}, X_{2} X_{3}, X_{2} X_{4}\right]$. The corresponding affine variety $W:=\mathbb{C}^{4} / / \mathbb{C}^{*}$ is the hypersurface $\left\{Y_{1} Y_{2}-Y_{3} Y_{4}=0\right\} \subset \mathbb{C}^{4}$. Clearly $W$ is homogeneous and has an isolated singular point at the vertex $w$. The inverse image of $w$ in $\mathbb{C}^{4}$ has codimension 2 . Hence $\pi_{1}^{w}(W)=(1)$. It is easy to see that $H_{2}^{w}(W)$ has rank $>0$. Further, $\mathbb{C}\left[X_{1} X_{3}, X_{1} X_{4}, X_{2} X_{3}, X_{2} X_{4}\right]$ has infinite cyclic divisor group.

It is proved in [18] that the affine 3 -fold $W^{\prime}$ given by

$$
\left\{Y_{1} Y_{2}-Y_{3} Y_{4}+F\left(Y_{1}, Y_{2}, Y_{3}, Y_{4}\right)=0\right\}
$$

in $\mathbb{C}^{4}$ is a UFD if $F$ is a sufficiently general homogeneous polynomial of large degree. The analytic type of the singularity of $W^{\prime}$ at the origin is the same is that of $W$, hence a rational singularity. The local ring of $W^{\prime}$ at the origin is a UFD but its completion has infinite divisor class group.

This example illustrates that the hypothesis on $G^{0}$ in the theorem is necessary.

\section{References}

[1] V. I. Arnold, Local normal forms of functions. Invent. Math. 35 (1976), 87-109. Zbl 0336.57022 MR 0467795

[2] J. F. Boutot, Singularités rationelles et quotients par les groupes rèductifs. Invent. Math. 88 (1987), 65-68. Zbl 0619.14029 MR 0877006 
[3] H. Flenner, Die Sätze von Bertini für lokale Ringe. Math. Ann. 229 (1977), 97-111. Zbl 0398.13013 MR 0460317

[4] H. Flenner, Divisorenklassengruppen quasihomogener Singularitäten. J. Reine Angew. Math. 328 (1981), 128-160. Zbl 0457.14001 MR 0636200

[5] T. Fujita, On the topology of noncomplete algebraic surfaces. J. Fac. Sci. Univ. Tokyo, Sect. IA Math. 29 (1982), 503-566. Zbl 0513.14018 MR 0687591

[6] B. Giesecke, Simpliziale Zerlegung abzählbarer analytischer Räume. Math. Z. 83 (1964), 177-213. Zbl 0123.39602 MR 0159346

[7] M. Goresky and R. MacPherson, Stratified Morse Theory. Ergeb. Math. Grenzgeb. 14, Springer-Verlag, Berlin 1988. Zbl 0639.14012 MR 0932724

[8] R. V. Gurjar, On a conjecture of C. T. C. Wall. J. Math. Kyoto Univ. 31 (1991), 1121-1124. Zbl 0784.14023 MR 1141088

[9] R. V. Gurjar, Two-dimensional quotients of $\mathbb{C}^{n}$ are isomorphic to $\mathbb{C}^{2} / \Gamma$. Transform. Groups 12 (2007), 117-125. Zbl 1122.32015 MR 2308031

[10] H. Hamm and Lê Dũng Tráng, Lefschetz theorems on quasiprojective varieties. Bull. Soc. Math. France 113 (1985), 123-142. Zbl 0602.14009 MR 0820315

[11] H. Hamm and Lê Dũng Tráng, Local generalizations of Lefschetz-Zariski theorems. J. Reine Angew. Math. 389 (1988), 157-189. Zbl 0646.14012 MR 0953670

[12] H. Hamm and Lê Dũng Tráng, Relative homological depth and a conjecture of Deligne. Math. Ann. 296 (1993), 87-101. Zbl 0795.32014 MR 1213373

[13] G. Kempf, Some quotient surfaces are smooth. Michigan Math. J. 24 (1977), 347-352. Zbl 0385.14016 MR 0491675

[14] H. P. Kraft, T. Petrie and J. Randall, Quotient varieties. Adv. Math. 74 (1989), 145-162. Zbl 0691.14029 MR 0997095

[15] H. P. Kraft and P. Russell, G-bundles and linearization. Preprint, 2002.

[16] D. Luna, Slices étales. In Sur les groupes algébriques, Memoire 33, Suppl. Bull. Soc. Math. France, Soc. Math. France, Paris 1973, 81-105. Zbl 0286.14014 MR 0342523

[17] A. T. Lundell and S. Weingram, The Topology of CW Complexes. Van Nostrand Reinhold, New York 1969. Zbl 0207.21704

[18] A. J. Parameswaran and V. Srinivas, A variant of the Noether-Lefschetz theorem: Some new examples of unique factorization domains. J. Algebraic Geom. 3 (1994), 81-115. Zbl 0814.13013 MR 1242007

[19] M. Reid, Canonical 3-folds. In Géométrie Algébrique Angers 1979 (A. Beauville, ed.), 1980, Sijthoff \& Noordhoff, Alphen aan den Rijn, The Netherlands, 273-310. Zbl 0451.14014 MR 0605348

[20] E. H. Spanier, Algebraic Topology. McGraw-Hill, New York 1966. Zbl 0145.43303 MR 0210112

Received December 7, 2007

R. V. Gurjar, School of Mathematics, Tata Institute of Fundamental Research, Homi-Bhabha road, Mumbai 400005, India

E-mail: gurjar@math.tifr.res.in 